\section{Un nouveau rôle pour le canal ionique TRPV 1 dans I'activation des lymphocytes T CD4}

Samuel Bertin, Eyal Raz
University of California, San Diego, Department of Medicine, Stein Clinical Research, Room 126, 9500 Gilman Drive, La Jolla, CA 92093-0663,

États-Unis.

sbertin@ucsd.edu
> Les ions calcium $\left(\mathrm{Ca}^{2+}\right)$ régulent une multitude de processus cellulaires, et la signalisation induite par le $\mathrm{Ca}^{2+}$ est essentielle à la fonction de la plupart des cellules, y compris les lymphocytes T CD4 (LT CD4) [1]. Ces derniers orchestrent la réponse immunitaire à médiation cellulaire à l'encontre de potentiels agents pathogènes, de cellules infectées par des virus ou de cellules tumorales, et sont également impliqués dans la réponse inflammatoire associée à certaines maladies auto-immunes telles que les maladies inflammatoires chroniques de l'intestin ( $\mathrm{MICl}$ ) [2]. Une entrée de $\mathrm{Ca}^{2+}$ est un événement essentiel à l'activation des LT CD4 et à leur différenciation en lymphocytes $T$ effecteurs [1]. Bien que les canaux CRAC $\left(\mathrm{Ca}^{2+}\right.$-release activated $\mathrm{Ca}^{2+}$ channel) soient reconnus comme importants pour ce processus, de nombreuses études indiquent que les LT CD4 expriment également d'autres canaux ioniques dont les fonctions sont, pour la plupart, méconnues [3, 4].

\section{Activation des lymphocytes T parle $\mathrm{Ca}^{2+}$}

Jusqu'à ce jour, la recherche sur l'entrée de $\mathrm{Ca}^{2+}$ dans les cellules non excitables a été dominée par le concept d'entrée de $\mathrm{Ca}^{2+}$ dépendante de la libération des réserves (SOCE ou store operated $\mathrm{Ca}^{2+}$ entry) [1, 3]. Dans ce modèle, l'entrée de $\mathrm{Ca}^{2+}$ se fait en deux temps. Ainsi, la libération des réserves calciques intracellulaires (par exemple les citernes du réticulum endoplasmique et l'appareil de Golgi) précède et induit l'entrée de $\mathrm{Ca}^{2+}$ extracellulaire par des canaux calciques localisés sur la membrane plasmique. Des travaux menés au cours des 10 dernières années ont permis d'identifier les protéines ORAll et STIMI (stromal interacting molecule 1) comme les constituants principaux des canaux CRAC et largement responsables de l'influx SOCE [1, 3, 5]. Le rôle important de ces canaux a notamment été reconnu lors de l'identification de mutations, entre autres dans les gènes ORAII et STIMI, dans le déficit immunitaire combiné sévère (DICS) [5] $(\rightarrow)$.

$(\rightarrow)$ Voir la Synthèse de F. Le Deist et T. Capiod, $m / s n^{\circ} 8-9$, août-septembre 2011, page 737

Bien que le rôle des

canaux CRAC et de l'entrée SOCE dans l'activation lymphocytaire soit incontestable, il existe également une autre voie d'entrée de $\mathrm{Ca}^{2+}$ dont l'activation est totalement indépendante de la libération des réserves calciques intracellulaires (SICE store independent $\mathrm{Ca}^{2+}$ entry) $[1,3]$. Cependant, ce type d'entrée de $\mathrm{Ca}^{2+}$, ainsi que les acteurs moléculaires en jeu, n'ont à ce jour pas encore été clairement identifiés. Parmi les canaux ioniques potentiellement responsables de I'influx SICE, certains canaux de la famille $(\rightarrow)$ Voir la Synthèse TRP (transient receptor potential) ont été évoqués [6] $(\rightarrow)$. de G. Vassort et J. Fauconnier, $m / s$ $n^{\circ} 2$, février 2008, page 163

Le rôle du canal ionique TRPV1 TRPVI (transient receptor potential vanilloid-1) a été le premier canal de la famille TRP à être identié chez les mammifères. Il est aussi connu sous le nom de récepteur de la capsaïcine, l'ingrédient actif trouvé dans les piments forts [7]. Depuis sa découverte, TRPVI a principalement été étudié dans le système nerveux périphé- rique et il a été établi que ce canal joue un rôle prépondérant dans la perception de la douleur. Cependant, il est très exprimé dans le corps en dehors des systèmes nerveux central et périphérique, par exemple dans la peau, l'appareil digestif, et le système immunitaire $[6,8]$. Ainsi, nous avons récemment découvert l'expression des canaux TRPVI à la surface des LT CD4 et leur rôle dans l'entrée de $\mathrm{Ca}^{2+}$ dans ces cellules dans des conditions physiologique et pathologique [9].

À l'aide de techniques d'imagerie calcique, nous avons démontré que la capsaïcine, un agoniste sélectif du canal TRPV 1 [7], induit un influx de $\mathrm{Ca}^{2+}$ extracellulaire dans les LT CD4 isolés de souris sauvages, mais pas dans les LT CD4 isolés de souris déficientes pour TRPV1. Nous avons ensuite réalisé des expériences de patch-clamp et confirmé les caractéristiques biophysiques des canaux TRPVl exprimés à la surface des LT CD4. Bien que le rapport de perméabilité au $\mathrm{Ca}^{2+}$ et au sodium $\left(\mathrm{P}_{\mathrm{Ca}} / \mathrm{P}_{\mathrm{Na}}\right)$ soit d'environ 10 pour TRPV1 [7], et donc par comparaison moins sélectif pour le $\mathrm{Ca}^{2+}$ que ce rapport dans les canaux CRAC [3, 4], nous avons identifié que l'influx de $\mathrm{Ca}^{2+}$ induit par le TCR ( $T$ cell receptor) était moindre dans les LT CD4 déficients pour TRPVI que dans les LT CD4 de type sauvage. Cette observation était également reproductible après traitement de LT CD4 sauvages par des antagonistes sélectifs pour TRPVl. En revanche, une activation des LT CD4 de façon indépendante du TCR (T-cell receptor) - avec I'ionomycine ou la thapsigargine (un inhibiteur spécifique des ATPases-Ca ${ }^{2+}$ SERCA qui pompent le $\mathrm{Ca}^{2+}$ à l'intérieur 


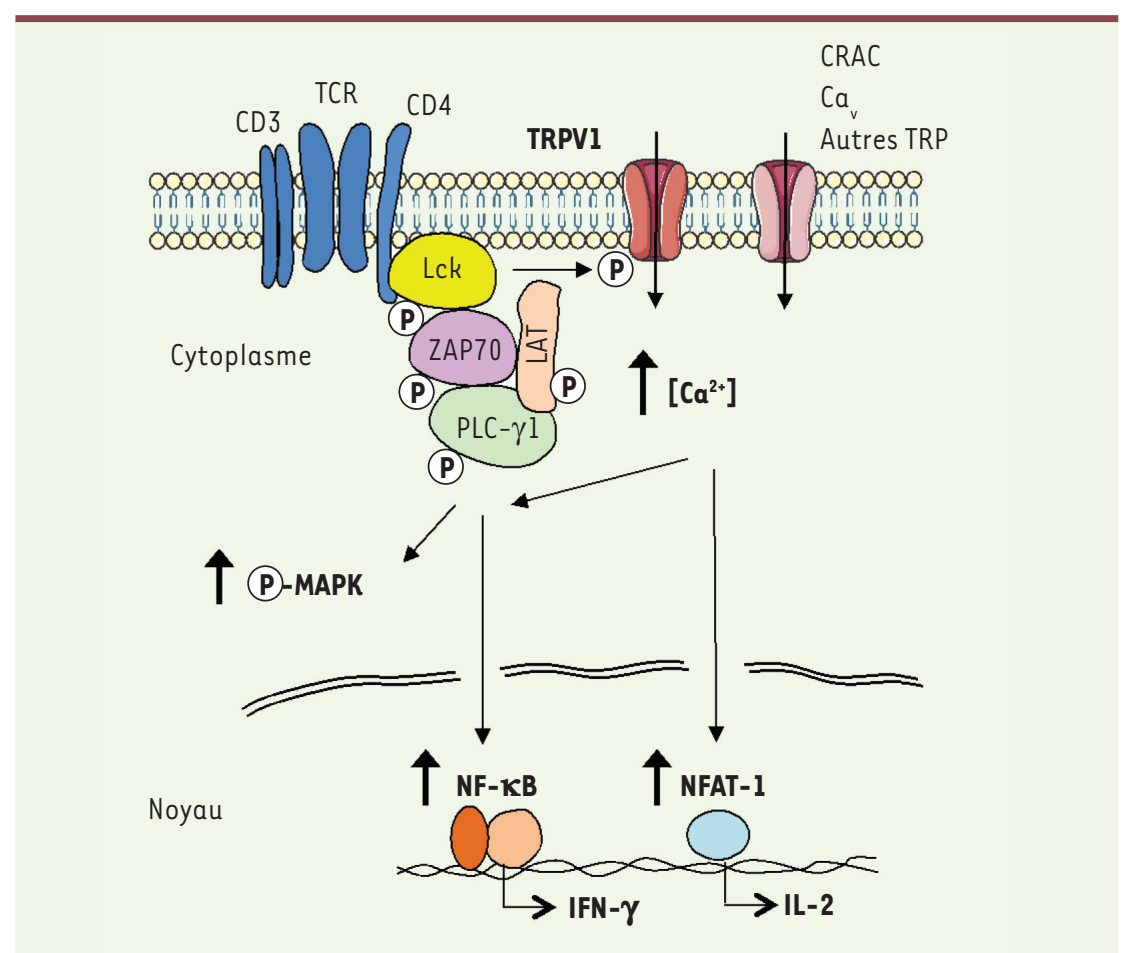

Figure 1. Modèle proposé pour la régulation de l'activation des LT CD4 par TRPV1. La stimulation du TCR induit une cascade de phosphorylations qui conduit à l'activation de canaux calciques localisés sur la membrane plasmique. Nos observations suggèrent que TRPVl fait partie de ces canaux et contribue à l'influx de $\mathrm{Ca}^{2+}$ nécessaire à l'activation de voies de signalisation en aval ainsi qu'aux fonctions des LT-CD4, comme par exemple la production de cytokines. (c) : Nature publishing group [9].

du réticulum) - n'affectait pas l'influx de $\mathrm{Ca}^{2+}$ via TRPVI. Ces résultats suggéraient que TRPVl agit comme un canal SICE dans les LT CD4 et contribue à l'influx de $\mathrm{Ca}^{2+}$ induit par le TCR.

Nous avons ensuite cherché à comprendre comment TRPVl est activé après stimulation du TCR. À l'aide de techniques d'imagerie confocale, nous avons constaté que TRPVl est physiquement associé à la molécule CD4 (un corécepteur du TCR) ainsi qu'à la protéine tyrosine kinase Lck dans les LT CD4. De plus, la stimulation du TCR induit rapidement la phosphorylation de TRPVI sur des résidus tyrosine, mais ce phénomène est absent dans des LT CD4 déficients en Lck. Sur la base de ces observations, nous avons postulé que la phosphorylation de TRPVI par Lck pourrait conduire à l'activation des canaux TRPVI après stimulation du TCR. Cependant, d'autres mécanismes en aval du TCR peuvent également contribuer à l'activation de TRPVl, telle que l'hydro- essentiel pour mieux comprendre les mécanismes conduisant à l'activation de ces cellules, et pour concevoir des stratégies thérapeutiques ciblant cette voie de signalisation, par exemple pour le traitement de maladies auto-immunes ou inflammatoires. Nos observations suggèrent que les canaux TRPVI jouent des rôles physiologiques importants au-delà de leurs fonctions largement décrites de récepteurs de la douleur dans les neurones sensoriels. Ainsi, notre étude démontre que l'influx de $\mathrm{Ca}^{2+}$ au travers des canaux TRPVl contribue à l'activation des LT CD4 et à la production de cytokines pro-inflammatoires par ces cellules (Figure 1). Ces données suggèrent que cibler TRPVI pourrait représenter une nouvelle stratégie thérapeutique pour inhiber les réponses pro-inflammatoires associées à certaines maladies autoimmunes telles que les MICI. $\diamond$

A novel role for the TRPVI ion channel in CD4 T cell activation

\section{LIENS D'INTÉRÊT}

Les auteurs déclarent n'avoir aucun lien d'intérêt concernant les données publiées dans cet article.

\section{RÉFÉRENCES}

phate (PIP2) par la phospholipase C- $\gamma 1$ (PLC- $\gamma 1)$, qui libère TRPVI de l'inhibition exercée par PIP2 [10].

Dans ces LT CD4 déficients en TRPVI, et contrairement aux LT-CD4 de type sauvage, nous avons observé une diminution de l'activation des facteurs de transcription NFAT (Nuclear factor of activated T-cells) et NF-kB, en accord avec la réduction de l'influx calcique induit par le TCR, ainsi qu'une réduction de la production de certaines cytokines, telles que l'interleukine-2 (IL-2) et I'interféron- $\gamma($ IFN- $\gamma)$, après stimulation du TCR. De plus, dans des modèles animaux de $\mathrm{MICl}$, la délétion génétique ou l'inhibition pharmacologique de TRPVl dans les LT CD4 réduisent de manière significative la gravité de la colite.

\section{Conclusions et perspectives}

Déterminer comment les signaux calciques sont régulés dans les $\mathrm{LT}$ CD4 est
1. Hogan PG, Lewis RS, Rao A. Molecular basis of calcium signaling in lymphocytes: STIM and ORAI. Annu Rev Immunol $2010 ; 28$ : 491-533.

2. Monteleone G, Caprioli F. T-cell-directed therapies in inflammatory bowel diseases. Clin Sci (Lond) 2010 ; $118: 707-15$

3. Feske S, Skolnik Ey, Prakriya M. Ion channels and transporters in lymphocyte function and immunity. Nat Rev Immunol $2012 ; 12$ : 532-47.

4. Feske $\mathrm{S}$. $\mathrm{Ca}^{2+}$ influx in T cells : how many $\mathrm{Ca}^{2+}$ channels? Front Immunol $2013 ; 4: 99$.

5. Le Deist F, Capiod T. Immunodéficiences et pathologies associées aux mutations dans STIM/ORAI : un complexe membranaire au cœur de la signalisation calcique. Med Sci (Paris) 2011 ; 27 : 737-45.

6. Vassort $G$, Fauconnier ). Les canaux TRP (transient receptor potential) : une nouvelle famille de canaux à expression variée. Med Sci (Paris) 2008 ; 24 : 163-8.

7. Caterina MJ, Schumacher MA, Tominaga M, et al. The capsaicin receptor : a heat-activated ion channel in the pain pathway. Nature $1997 ; 389: 816-24$.

8. Fernandes $\varepsilon S$, Fernandes MA, Keeble JE. The functions of TRPAl and TRPVl : moving away from sensory nerves. BrJ Pharmacol $2012 ; 166: 510-21$.

9. Bertin S, Aoki-Nonaka Y, de Jong PR, et al. The ion channel TRPVI regulates the activation and proinflammatory properties of $\mathrm{CD}^{+} \mathrm{T}$ cells. Nat Immunol 2014 ; 15 : 1055-63.

10. Prescott $\varepsilon D$, Julius D. A modular PIP2 binding site as a determinant of capsaicin receptor sensitivity. Science $2003 ; 300: 1284-8$. 